Pacific Journal of Mathematics

STRONGLY SUPERFICIAL ELEMENTS 


\section{STRONGLY SUPERFICIAL ELEMENTS}

\section{Merriline Smith}

The concepts of a strongly superficial element and a very strongly superficial element are introduced. A number of their properties are established and three applications are given.

1. Introduction. Superficial elements have proved to be a useful and important concept in a number of problems in commutative algebra, for example, the study of characteristic functions and multiplicities. This paper is concerned with two special kinds of such elements: a very strongly superficial (v.s.s.) element of degree $k$ for an ideal $A$ in a ring $R$; and, a strongly superficial (s.s.) element for $A^{k}$. After listing a number of properties of s.s. and v.s.s. elements, we present in Theorem (2.5) and (2.6) a number of characterizations of such elements. In $\$ 3$ we give three applications of the theorems. Namely, we first show that a known result about s.s. elements for an ideal generated by an $R$-sequence in a locally Macaulay ring holds in every Noetherian ring (3.2). Next we show that if $A$ is an ideal in a Noetherian ring $R$, then the zero ideal in the $A$-form ring of $R$ has no irrelevant prime divisor if and only if there exists a v.s.s. element of some positive degree for $A$ (3.5). The final application is concerned with certain ideals in Rees rings of $R$ ((3.8) and (3.9)).

2. s.s. and v.s.s. elements. All rings in this paper are assumed to be commutative with a unit element.

Definition. 2.1. Let $A$ be an ideal in a ring $R$, and let $k$ be a positive integer. A superficial element of degree $k$ for $A$ is an element $x \in A^{k}$ for which there exists a nonnegative integer $c$ such that $\left(A^{n+k}: x R\right) \cap A^{c}=A^{n}$, for all integers $n \geqq c$. If $c=0$ (where $A^{0}=R$ ), then $x$ is said to be a very strongly superficial (v.s.s.) element of degree $k$ for $a$. If $A^{n k}: x R=A^{n k-k}$, for all integers $n \geqq 1$, then $x$ is said to be a strongly superficial (s.s) element for $A^{k}$.

It is easily seen that, if $A^{n} \neq A^{n+1}$ (for each integer $n \geqq 0$ ) and $x$ is a superficial element of degree $k$ for $A$, then $x \notin A^{k+1}$. (In particular, a v.s.s. element of degree $k$ for $A$ is not in $A^{k+1}$.) It is also clear that a v.s.s. element of degree $k$ for $A$ is a s.s. element for $A^{k}$. Some further properties of such elements are given in the following remark.

Remark 2.2. Let $A$ be an ideal in a Noetherian ring $R$, let $k$ be a positive integer, and assume $x$ is a s.s. element for $A^{k}$. 
(2.2.1) If $k=1$, then $x$ is a v.s.s. element of degree 1 for $A$, so the concepts of a s.s. element for $A$ and of a v.s.s. element of degree 1 for $A$ are the same.

(2.2.2) If $y$ is a v.s.s. element of degree $n$ for $A$, where $n=m k$, for some positive integer $m$, then it is readily seen that $x y$ is a s.s. element for $A^{n+k}$. Also, $x^{n}$ is a s.s. element for $A^{n k}$, for each integer $n \geqq 1$.

(2.2.3) If $x y=0$, for some $y \in R$, then $y \in \bigcap_{n \geqq 1}\left(A^{n k}: x R\right)=$ $\bigcap_{n \geqq 1} A^{n k-k}$. Therefore, if $\bigcap_{n \geqq 1} A^{n}=(0)$, then a s.s. element is not a zero-divisor.

(2.2.4) If $x$ is a v.s.s. element of degree $k$ for $A$, then statements analogous to (2.2.2)-(2.2.3) hold.

Theorems (2.5) and (2.6) below give several necessary and sufficient conditions for $x$ to be a v.s.s. element of degree $k$ for $A$ (respectively, a s.s. element for $A^{k}$ ). To prove these results, the following lemma and definitions are needed.

Lemma 2.3. Let $A$ be an ideal in a ring $R$, let $x \in A^{k}$, and assume $x$ is a nonzero-divisor in $R$. Then $A^{n k}: x R=A^{n k-k}$, for all $n \geqq 1$, if and only if $x^{n} R\left[A^{k} / x\right] \cap R=A^{n k}$, for all integers $n \geqq 1$.

Proof. Let $R^{\prime}=R\left[A^{k} / x\right]$. To show the "only if" part, fix an integer $n \geqq 1$. Since $x^{n} R^{\prime} \cap R \supseteq A^{k n}$, let $r \in x^{n} R^{\prime} \cap R$. Then $r=$ $x^{n} r^{\prime}$, for some $r^{\prime}=a / x^{j}$, where $a \in A^{k j}$, so $r x^{j}=x^{n} a \in A^{(n+j) k}$. Therefore, $r \in A^{(n+j) k}: x^{j} R=A^{n k}$. To see the "if" part, let $r \in A^{n k}: x R$. Then $\quad r x=a \in A^{n k}$. Hence $\quad r=x^{n-1}\left(a / x^{n}\right) \in x^{n-1} R^{\prime} \cap R=$ $A^{(n-1) k}$. The opposite containment is always true, because $x \in A^{k}$.

Definition. 2.4. Let $A=\left(a_{1}, \cdots, a_{n}\right)$ be an ideal in a Noetherian ring $\mathscr{R}$, let $u$ be an indeterminate, and let $t=u^{-1}$.

(2.4.1) The graded Noetherian ring $\mathscr{R}=\mathscr{R}(R, A)=$ $R\left[t a_{1}, \cdots, t a_{n}, u\right]$ is the Rees ring of $R$ with respect to $A$. The elements of $R$ in $t^{r} A^{r}$ are said to be homogeneous elements of degree $r$ $\left(-\infty<r<\infty\right.$, where $A^{r}=R$ if $\left.r \leq 0\right)$ and a homogeneous ideal is an ideal which can be generated by homogeneous elements. A homogeneous ideal $H$ in $\mathscr{R}$ is said to be irrelevant in case it contains every homogeneous element of sufficiently large degree. Otherwise, $H$ is said to be relevant.

(2.4.2) The graded subring $\mathscr{S}=\mathscr{S}(R, A)=R\left[t a_{1}, \cdots, t a_{n}\right]$ of $\mathscr{R}$ is the restricted Rees ring of $R$ with respect to $A$. 
(2.4.3) The form ring of $R$ with respect to $A$ (or, $A$-form ring of $R$ ), $\mathscr{F}(R, A)$, is the graded ring $\bigoplus_{i=0}^{\infty} A^{i} / A^{i+1}$. It is known [3, Theorem 2.1] that $\mathscr{F}=\mathscr{F}(R, A) \cong \mathscr{R} / u \mathscr{R}$, where $\mathscr{R}=\mathscr{R}(R, A)$, and in this isomorphism the $A$-form of an element $x$ in $R$ corresponds to the coset $x t^{k}+u \mathscr{R}$ in $\mathscr{R} / u \mathscr{R}$. (The assumption in [3] that $R$ be local is not essential.)

THEOREM. 2.5. Let $A$ be an ideal in a Noetherian ring $R$, let $\mathscr{R}=\mathscr{R}(R, A)$, and let $\mathscr{S}=\mathscr{S}(R, A)$. Fix a positive integer $k$, fix $x \in A^{k}$, and consider the following statements:

(i) $\quad x$ is a v.s.s. element of degree $k$ for $A$.

(ii) $\quad x$ is a v.s.s. element of degree $k$ for $A \mathscr{S}$ in $\mathscr{S}$.

(iii) $\quad x$ is a v.s.s. element of degree $k$ for $u \mathscr{R}$ in $\mathscr{R}$.

(iv) $x t^{k}$ is not in any prime divisor of $A^{i} \mathscr{Y}$, for all $i \geqq 1$.

(v) $\quad u, x t^{k}$ is an $\mathscr{R}$-sequence.

(vi) $\quad A^{n+k} \cap x R=x A^{n}$, for every integer $n \geqq 1$.

(vii) $A^{n+k} \mathscr{S} \cap x \mathscr{S}=x A^{n} \mathscr{S}$, for every integer $n \geqq 1$.

(viii) $u^{n+k} \mathscr{R} \cap x \mathscr{R}=\left(x u^{n}\right) \mathscr{R}$, for every integer $n \geqq 1$.

(ix) $\quad x$ is a nonzero-divisor and $x^{n} R^{\prime} \cap R=A^{n k}$, for every integer $n \geqq 1$, where $R^{\prime}=R\left[A^{k} / x\right]$.

Then the following hold:

(2.5.1) (i)-(v) are equivalent and each implies (vi)-(viii).

(2.5.2) (vi)-(viii) are equivalent and, if $x$ is a nonzero-divisor, then each implies (i)-(v) and (ix).

(2.5.3) If $k=1$, then (ix) implies (i)-(viii).

Proof. (i) $\rightarrow$ (iii). $\quad x=u^{k}\left(x t^{k}\right) \in u^{k} \mathscr{R}, \quad$ and $\quad u^{n+k} \mathscr{R}: x \mathscr{R}=$ $u^{n} \mathscr{R}: x t^{k} \mathscr{R} \supseteq u^{n} \mathscr{R}$. For the opposite inclusion, let $y t^{r} \in$ $u^{n+k} \mathscr{R}: x \mathscr{R}$. Then, with $m=n+k+r$, there exists $a \in A^{m}$ such that $x y t^{r}=u^{n+k} a t^{m}$. Therefore, $x y=a \in A^{m}$; hence $y \in A^{n+r+k}: x R=$ $A^{n+r}$, by (i). Therefore, $y t^{n+r} \in \mathscr{R}$, so $y t^{r} \in u^{n} \mathscr{R}$. Hence, since $u^{n} \mathscr{R}: x \mathscr{R}$ is homogeneous, (iii) holds.

(iii) implies $\quad u \mathscr{R}=u^{k+1} \mathscr{R}: x \mathscr{R}=u^{k+1} \mathscr{R}: u^{k}\left(x t^{k}\right) \mathscr{R}=$ $\left(u^{k+1} \mathscr{R}: u^{k} \mathscr{R}\right): x t^{k} \mathscr{R}=u \mathscr{R}: x t^{k} \mathscr{R}$. Hence (iii) implies (v), since $u$ is not a zero-divisor in $\mathscr{R}$.

(v) $\rightarrow$ (iv). Let $i \geqq 1$ and let $a t^{n} \in A^{i} \mathscr{S}: x t^{k} \mathscr{S}$. Then $a t^{n} x t^{k} \in$ $A^{i} \mathscr{S}=u^{i} \mathscr{R} \cap \mathscr{S}$ (this can be seen much as in the remainder of this paragraph). Hence $a t^{n} \in u^{i} \mathscr{R}: x t^{k} \mathscr{R}=\left(\right.$ by (v)) $u^{i} \mathscr{R}$, and so $a t^{n+i} \in \mathscr{R}$, thus $a \in A^{n+i}$. Therefore $a=\Sigma b_{g} c_{g}$, where $b_{g} \in A^{i}$ and $c_{g} \in A^{n}$, hence $a t^{n}=\sum b_{g}\left(c_{g} t^{n}\right) \in A^{i} \mathscr{Y}$, and so (iv) holds.

(iv) $\rightarrow$ (ii). Since $A^{i \mathscr{S}}: x \mathscr{S} \supseteq A^{i-k} \mathscr{Y}$, for all $i \geqq k$, and both ideals are homogeneous, let $y t^{r}$ be an arbitrary homogeneous element in $A^{i} \mathscr{Y}: x \mathscr{S}$. Then $x y t^{r} \in A^{i-k} A^{k} \mathscr{Y}$, say $x y t^{r}=\Sigma_{g, j} a_{g, j} b_{g, j}\left(c_{g, j} t^{r}\right)$, where 
each $a_{g, j} \in A^{i-k}, b_{g, j} \in A^{k}$, and $c_{g, j} t^{r} \in \mathscr{S}$, so $x t^{k} y t^{r}=\Sigma_{g, j} a_{g, j}\left(b_{g, j} t^{k}\right)\left(c_{g, j} t^{r}\right)$, where each $a_{g, j} \in A^{i-k}$ and $b_{g, j} c_{g, j} t^{r+k} \in \mathscr{S}$. Hence $x t^{k} y t^{r} \in A^{i-k} \mathscr{Y}$, so that $y t^{r} \in A^{i-k} \mathscr{Y}$, by (iv), and so (ii) holds.

Since $\mathscr{S} \subseteq R[t]$, (ii) implies, for all $i \geqq k, A^{i-k}=A^{i-k} \mathscr{S} \cap R=$ $\left(A^{i} \mathscr{S}: x \mathscr{S}\right) \cap R=\left(A^{i} \mathscr{S} \cap R\right):(x \mathscr{S} \cap R)=A^{i} R: x R$, hence (ii) implies (i). Therefore, (i)-(v) are equivalent, and if (i) holds, then

(*) $\quad A^{n+k} \cap x R=x\left(A^{n+k}: x R\right)=x A^{n}$, for all $n \geqq 1$, hence (i) implies (vi). Similarly (ii) implies (vii) and (iii) implies (viii). Therefore, (2.5.1) holds.

(2.5.2) (viii) $\rightarrow$ (vii) much as in the proof that (v) $\rightarrow$ (iv); and (vii) $\rightarrow$ (vi), since $\mathscr{S} \subseteq R[t]$. For (vi) $\rightarrow$ (viii), let $a t^{i} \in u^{n+k} \mathscr{R} \cap x \mathscr{R}=$ $x\left(u^{n+k} \mathscr{R}: x \mathscr{R}\right), \quad$ so $a t^{i}=x b t^{i} \in u^{n+k} \mathscr{R}$, for some $b t^{i} \in$ $u^{n+k} \mathscr{R}: x \mathscr{R}$. Therefore, with $g=n+i+k, \quad a=b x \in A^{g}$, so $b \in A^{s}: x R$, hence $\quad x b \in x\left(A^{s}: x R\right)=A^{g} \cap x R=$ (by $\quad$ (vi)) $x A^{n+i}$. Therefore $x b=\sum x c_{f} d_{f}$, where $x c_{f} \in x A^{n}$ and $d_{f} \in A^{i}$, hence $a t^{i}=x b t^{i} \in x A^{n} \mathscr{R} \subseteq x u^{n} \mathscr{R}$, and so $u^{n+k} \mathscr{R} \cap x \mathscr{R}=x\left(u^{n+k} \mathscr{R}: x \mathscr{R}\right) \subseteq$ $x u^{n} \mathscr{R}$, since $u^{n+k} \mathscr{R} \cap x \mathscr{R}$ is homogeneous. Hence (viii) holds, since $x u^{n} \mathscr{R} \subseteq u^{n} \mathscr{R}$ and since $x \in A^{k}$ implies $u^{n+k} \mathscr{R}: x \mathscr{R} \supseteq u^{n} \mathscr{R}$. Further, if $x$ is a nonzero-divisor, then (vi) implies (i), by (*), and so (ix) holds, by (2.3).

Finally, for (2.5.3), if $k=1$, then (ix) implies (i), by (2.3).

Theorem 2.6. Let $R, A, x$ and $k$ be as in (2.5), let $\mathscr{R}=\mathscr{R}\left(R, A^{k}\right)$, let $\mathscr{S}=\mathscr{S}\left(R, A^{k}\right)$, and consider the following statements:

(i) $\quad x$ is a s.s. element for $A^{k}$.

(ii) $\quad x$ is a s.s. element for $A^{k} \mathscr{Y}$.

(iii) $\quad x$ is a s.s. element for $u \mathscr{R}$.

(iv) $x t$ is not in any prime divisor of $A^{k i} \mathscr{Y}$, for each $i \geqq 1$.

(v) $\quad u, x t$ is an $\mathscr{R}$-sequence.

(vi) $\quad A^{n k} \cap x R=x A^{n k-k}$, for all integers $n \geqq 1$.

(vii) $\quad A^{n k} \mathscr{S} \cap x \mathscr{S}=x A^{n k-k} \mathscr{S}$, for all integers $n \geqq 1$.

(viii) $u^{n} \mathscr{R} \cap x \mathscr{R}=x u^{n-1} \mathscr{R}$, for all integers $n \geqq 1$.

(ix) $\quad x$ is a nonzero divisor and $x^{n} R^{\prime} \cap R=A^{\text {nk }}$, for all integers $n \geqq 1$, where $R^{\prime}=R\left[A^{k} / x\right]$.

Then the following statements hold:

(2.6.1) (i)-(v) are equivalent and each implies (vi)-(viii).

(2.6.2) (vi)-(viii) are equivalent and, if $x$ is not a zero divisor, then each implies (i)-(v) and (ix).

(2.6.3) (ix) implies (i)-(viii).

Proof. This follows from (2.2.1) and (2.5). 
3. Applications. In this section we give three applications of Theorems (2.5) and (2.6).

Remark. 3.1. Let $a_{1}, \cdots, a_{m}$ be an $R$-sequence in a Noetherian ring $R$, and let $A=\left(a_{1}, \cdots, a_{m}\right) R$.

(3.1.1) [2, Corollary 3.7]. If $R$ is locally Macaulay, then $a_{1}$ is a s.s. element for $A$.

(3.1.2) If $R$ is a Macaulay local ring, then each of the following statements hold:

(i) ([5, Lemma 6, p. 402] and [1, Theorem 119].) Each $a_{i}$ is a s.s. element for $A$.

(ii) [5, Lemma 5, p. 401]. The prime divisors of $A^{n}(n \geqq 1)$ are the prime divisors of $A$ and each has height $m$.

We note that it follows from (3.1.2) that parts (i) and (ii) of (3.1.2) also hold for an ideal generated by an $R$-sequence in a locally Macaulay ring. However, it follows from (3.2) below that (3.1.2) (i) holds even if $R$ is not locally Macaulay.

Proposition $^{1}$ 3.2. Let $R$ be a Noetherian ring, let $a_{1}, \cdots, a_{m}$ be an $R$-sequence, and let $A=\left(a_{1}, \cdots, a_{m}\right) R$. Then $A^{n}: a_{i} R=A^{n-1}$, for every integer $n \geqq 1$ and for every $i=1, \cdots, m$.

1 I am grateful to the referee for mentioning that this result was proved in D. Taylor, "Ideals generated by monomials in an $R$-sequence," Thesis, University of Chicago, 1966 . Since her thesis isn't readily available, the referee kindly provided the following proof of a generalization of (3.2): Let $R$ be a commutative ring with identity, $M$ an $R$-module, $a_{1}, \cdots, a_{m}$ an $M$-sequence in $R$, and $A=\left(a_{1}, \cdots, a_{m}\right) R$. Then, for all positive integers $n$ and for $i=1, \cdots, m, A^{n} M: a_{1} R=A^{n-1} M$.

Proof. Let $S=Z\left[x_{1}, \cdots, x_{m}\right], I=\left(x_{1}, \cdots, x_{m}\right) S, \phi: S \rightarrow R$ by $\phi\left(x_{j}\right)=a_{j}$ (so that $R, M$ become $S$-modules) and consider the commutative diagram:

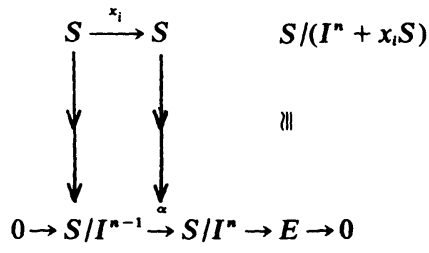

In order that $A^{n} M: a_{i} R=A^{n-1} M$ it suffices that the bottom row remain exact upon applying $\bigotimes_{R} M$.

Hence, it suffices that $\operatorname{Tor}_{1}^{s}(E, M)=0$. But $E$ is easily seen to have a filtration all of whose factors are $\cong F=S /\left(x_{1}, \cdots, x_{m}\right) S$ ( $F \cong Z$, of course). Thus, a sufficient condition for $A^{n} M: a_{i} R=A^{n-1} M$, all $n$, is that $\operatorname{Tor}_{1}^{S}(F, M)=0$, which is immediate if $a_{1}, \cdots, a_{m}$ is a regaular sequence on $M$. 
Proof. Let $\mathscr{R}=\mathscr{R}(R, A)$. By [4, Theorem 3.5.1], $a_{i} t$ is not in any prime divisor of $u \mathscr{R}$, for every $i=1, \cdots, m$. Hence, we are done by (2.5) (i) and (v).

Clearly, (3.2) and (2.5) show that, with $R, a_{1}, \cdots, a_{m}$ and $A$ as in (3.2), each $a_{i}$ is a s.s. element for $A \mathscr{S}$ in $\mathscr{S}=\mathscr{S}(R, A)$ and for $u \mathscr{R}$ in $\mathscr{R}=\mathscr{R}(R, A)$.

Definition 3.3. Let $A$ be an ideal in a Noetherian ring $R$. For all integers $s$, the $s$-component $H_{s}$ of a homogeneous ideal $H$ in $\mathscr{R}=$ $\mathscr{R}(R, A)$ is the ideal in $R, H_{s}=\left\{b \in R \mid t^{s} b \in H\right\}$.

It is easy to see that a homogeneous ideal $H$ in $\mathscr{R}$ is irrelevant if and only if $H_{s}=A^{s}$, for all (or, for some) sufficiently large s. Equivalently, $H$ is irrelevant if and only if $H \supseteq\left(A^{*}\right)^{s}=\left(A^{s}\right)^{*}$, for all (or, for some) sufficiently large $s$, where $B^{*}=B R[u, t] \cap \mathscr{R}$, for each ideal $B$ in $R$. (2.5) (v) shows that a sufficient condition for $u \mathscr{R}$ to have no irrelevant prime divisors is the existence of a v.s.s. element $x$ of some degree $k$ for $A$. That is, if $x t^{k}$ is not in any prime divisor $P$ of $u \mathscr{R}$, then clearly no power of $x t^{k}$ can belong to $P$. (3.4) below shows that the converse also holds.

THEOREM 3.4. Let $A$ be an ideal in a Noetherian ring $R$, and let $\mathscr{R}=\mathscr{R}(R, A)$. A necessary and sufficient condition for $u \mathscr{R}$ to have no irrelevant prime divisor is that there exists a v.s.s. element of some positive degree for $A$.

Proof. By the preceding discussion, it suffices to prove the "necessary part." Let $A^{*}=A R[u, t] \cap \mathscr{R}$, let $P_{1}, \cdots, P_{h}$ be the prime divisors of $u \mathscr{R}$, and let $N_{g}=\left\{c_{r} t^{r} ; c_{r} t^{r} \in P_{g}\right.$ and $\left.r \geqq 1\right\}$ be the set of all homogeneous elements of positive degree contained in $P_{g}$, for each $g=1, \cdots, h$. If we can find a homogeneous element of positive degree in $\mathscr{R}$ and not in any of the $N_{g}$, then we are done by (2.5) (i) and (v).

Since $P_{h}$ is relevant by hypothesis, $P_{h} \supseteq A^{*}$; therefore, there exists some $a \in A$ such that at $\notin N_{h}$. If at $\notin G=\bigcup_{g=1}^{h} N_{g}$, we are done. If at $\in G$, then, say, at $\in I=\bigcap_{i=1}^{m} N_{i}$ and at $\notin J=\bigcup_{j=m+1}^{h} N_{j}$. We can assume there are no containment relations among the $N_{g}$; thus $J^{\prime} \not I^{\prime}$, where $I^{\prime}=\bigcup_{i=1}^{m} N_{i}$ and $J^{\prime}=\bigcap_{j=m+1}^{h} N_{j}$. To see this, note that each homogeneous element in $N_{m+1} \cdots N_{h}$ is in $J^{\prime}$, because the $N_{j}$ are subsets of ideals. Therefore, if $J^{\prime} \subseteq I^{\prime}$, then $\left(N_{m+1} \cdots N_{h}\right) \mathscr{R} \subseteq \bigcup_{i=1}^{m} P_{i}$; hence there exists an $i(1 \leqq i \leqq m)$ and a $j(m+1 \leqq j \leqq h)$ such that $N_{j} \subseteq N_{i}$ which contradicts the assumption. Therefore, let $b t^{e}$ be a homogeneous element of positive degree such that $b t^{e} \in J^{\prime}$ and $b t^{e} \notin I^{\prime}$. Then $x t^{e}=(a t)^{e}+b t^{e}$ satisfies (2.5) (v). 
Corollary 3.5. Let $A$ be an ideal in a Noetherian ring $R$, and let $\mathscr{F}$ be the form ring of $R$ with respect to $A$. Then a necessary and sufficient condition for there to exist a v.s.s. element of some positive ddegree for $A$ is that the zero ideal in $\mathscr{F}$ has no irrelevant prime divisor.

(An irrelevant (homogeneous) ideal in $\mathscr{F}$ is defined in an analogous manner to (2.4.1).)

Proof. This follows immediately from (3.4) and the fact that $\mathscr{F} \cong \mathscr{R} / u \mathscr{R}[3$, Theorem 2.1], where $\mathscr{R}=\mathscr{R}(R, A)$.

Corollary 3.6. Let $A$ be an ideal in a Noetherian ring $R$, and assume there exists an element $x$ in $A$ such that $A^{m}: x R=A^{m-1}$, for all integers $m \geqq r$, where $r$ is some fixed positive integer. Then the following statements hold, for each integer $i \geqq r$ :

(3.6.1) $x^{i}$ is a v.s.s. element of degree one for $A^{i}$.

(3.6.2) If $M$ is a maximal ideal in $R$ such that $A \subseteq M$, then $u \mathscr{R}^{(i)}: \mathcal{M}_{i}=u \mathscr{R}^{(i)}, \quad$ where $\quad \mathscr{R}^{(i)}=\mathscr{R}\left(R, A^{i}\right), \quad$ and $\mathcal{M}_{i}=$ $\left(M R[u, t] \cap \mathscr{R}^{(i)}, u\right) \mathscr{R}^{(i)}$.

Proof. (3.6.1) is clear, because $\left(A^{i}\right)^{n+1}: x^{i} R=\left(A^{i n+i}: x R\right): x^{i-1} R=$ $\left(A^{i}\right)^{n}$, for all integers $n \geqq 1$. (3.6.2) follows from (3.6.1) and (3.4).

We conclude this paper with the following three observations.

Lemma 3.7. Let $A$ be an ideal in a ring $R$, and assume $x$ is an element in $R$ such that $A^{n}: x R=A^{n}$, for every integer $n \geqq 1$. If $(A, x) R \neq R$, then $x$ is a v.s.s. element of degree one for $(A, x) R$.

Proof.

$$
\begin{gathered}
(A, x)^{n} R: x R=\left(A^{n}, x(A, x)^{n-1}\right) R: x R \\
=A^{n}: x R+(A, x)^{n-1} R=A^{n}+(A, x)^{n-1}=(A, x)^{n-1},
\end{gathered}
$$

for every integer $n \geqq 1$.

Corollary 3.8. Assume $A$ is an ideal in a Noetherian ring $R$ containing $a$ v.s.s. element $x$ of degree $k$. Then $x t^{k}$ is a v.s.s. element of degree one for both $\left(u, x t^{k}\right) \mathscr{R}$ in $\mathscr{R}=\mathscr{R}(R, A)$ and $\left(A, x t^{k}\right) \mathscr{S}$ in $\mathscr{S}=$ $\mathscr{S}(R, A)$.

Proof. Clear by (3.7) and the equivalence of (2.5) (i), (iv), and (v). 
Let $A$ be an ideal in a Noetherian ring $R$, and let $\mathscr{R}=\mathscr{R}(R, A)$. It is easily shown (cf. [3]) that for every ideal $B$ in $R, B^{*}=B R[u, t] \cap \mathscr{R}$ is such that $B^{*}: u \mathscr{R}=B^{*}$, and $B^{* n} R[u, t]=B^{n} R[u, t]$, but it is not in general true that $B^{* n}=\left(B^{n}\right)^{*}$. However, it follows from considering homogeneous elements that $A^{* n}=\left(A^{n}\right)^{*}$, for each $n \geqq 1$.

Corollary 3.9. Let $A$ and $B$ be ideals in a Noetherian ring $R$ such that $A+B \neq R$, and let $\mathscr{R}=\mathscr{R}(R, A)$. If $\left(B^{*}\right)^{n}=\left(B^{n}\right)^{*}$, for each $n \geqq 1$, then $u$ is a v.s.s. element of degree one for $\left(B^{*}, u\right) \mathscr{R}$.

Proof. $\quad\left(B^{*}, u\right) \mathscr{R} \neq \mathscr{R}$, since $A+B \neq R$. Therefore (3.9) follows from (3.7), because $\left(B^{*}\right)^{n}: u \mathscr{R}=\left(B^{n}\right)^{*}: u \mathscr{R}=\left(B^{n}\right)^{*}=\left(B^{*}\right)^{n}$, for each $n \geqq 1$.

It is also clear, by the preceding discussion, that (3.9) holds, in particular, whenever $B=A$.

\section{REFERENCES}

1. I. Kaplansky, Commutative Rings, Boston: Allyn and Bacon, 1970.

2. L. J. Ratliff, Jr., Two notes on locally Macaulay rings, Trans. Amer. Math. soc., 119 (1965), 399-406.

3. D. Rees, A note on form rings and ideals, Matematika 4 (1957), 51-60.

4. V. M. Smith, On Monadic Transformations of Macaulay Rings, Thesis (Riverside, California ), 1972.

5. O. Zariski and P. Samuel, Commutative Algebra, Vol. II, New Jersey: D. Van Nostrand Company, Inc., 1960.

Received February 12, 1974. The results in this paper constitute part of the author's Ph.D. dissertation [4] at the University of California, Riverside, under Professor Louis J. Ratliff, Jr., to whom the author wishes to express her gratitutde for his encouragement and many helpful suggestions. 


\section{PACIFIC JOURNAL OF MATHEMATICS}

\section{EDITORS}

RICHARD ARENS (Managing Editor)

University of California

Los Angeles, California 90024

\section{J. DugundJI}

Department of Mathematics University of Southern California Los Angeles, California 90007

D. Gilbarg and J. Milgram

Stanford University

Stanford, California 94305

\section{ASSOCIATE EDITORS}
E. F. BECKENBACH
B. H. NeumanN
F. WoLF
K. YoshiDA

\section{SUPPORTING INSTITUTIONS}

\author{
UNIVERSITY OF BRITISH COLUMBIA \\ CALIFORNIA INSTITUTE OF TECHNOLOGY \\ UNIVERSITY OF CALIFORNIA \\ MONTANA STATE UNIVERSITY \\ UNIVERSITY OF NEVADA \\ NEW MEXICO STATE UNIVERSITY \\ OREGON STATE UNIVERSITY \\ UNIVERSITY OF OREGON \\ OSAKA UNIVERSITY
}

\author{
UNIVERSITY OF SOUTHERN CALIFORNIA \\ STANFORD UNIVERSITY \\ UNIVERSITY OF TOKYO \\ UNIVERSITY OF UTAH \\ WASHINGTON STATE UNIVERSITY \\ UNIVERSITY OF WASHINGTON \\ AMERICAN MATHEMATICAL SOCIETY
}

The Supporting Institutions listed above contribute to the cost of publication of this Journal, but they are not owners or publishers and have no responsibility for its contents or policies.

Mathematical papers intended for publication in the Pacific Journal of Mathematics should be in typed form or offset-reproduced (not dittoed), double spaced with large margins. Underline Greek letters in red, German in green, and script in blue. The first paragraph or two must be capable of being used separately as a synopsis of the entire paper. Items of the bibliography should not be cited there unless absolutely necessary, in which case they must be identified by author and Journal, rather than by item number. Manuscripts, in duplicate, may be sent to any one of the four editors. Please classify according to the scheme of Math. Reviews, Index to Vol. 39. All other communications should be addressed to the managing editor, or Elaine Barth, University of California, Los Angeles, California, 90024.

100 reprints are provided free for each article, only if page charges have been substantially paid. Additional copies may be obtained at cost in multiples of 50 .

The Pacific Journal of Mathematics is issued monthly as of January 1966. Regular subscription rate: $\$ 72.00$ a year (6 Vols., 12 issues). Special rate: $\$ 36.00$ a year to individual members of supporting institutions.

Subscriptions, orders for back numbers, and changes of address should be sent to Pacific Journal of Mathematics, 103 Highland Boulevard, Berkeley, California, 94708.

PUBLISHED BY PACIFIC JOURNAL OF MATHEMATICS, A NON-PROFIT CORPORATION Printed at Jerusalem Academic Press, POB 2390, Jerusalem, Israel.

$$
\begin{gathered}
\text { Copyright (C) } 1975 \text { Pacific Journal of Mathematics } \\
\text { All Rights Reserved }
\end{gathered}
$$




\section{Pacific Journal of Mathematics}

\section{Vol. 58, No. $2 \quad$ April, 1975}

Zvi Artstein and John Allen Burns, Integration of compact set-valued functions . . . . . . . . . 297

Mark Benard, Characters and Schur indices of the unitary reflection group $[321]^{3} \ldots \ldots \ldots . .309$

Simeon M. Berman, A new characterization of characteristic functions of absolutely continuous

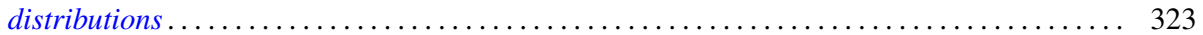

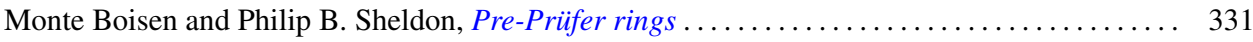

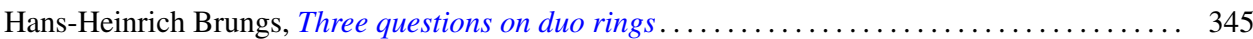

Iracema M. Bund, Birnbaum-Orlicz spaces of functions on groups................. 351

John D. Elwin and Donald R. Short, Branched immersions between 2-manifolds of higher

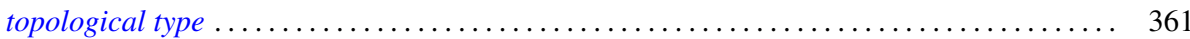

Eric Friedlander, Extension functions for rank 2, torsion free abelian groups . .......... 371

Jon Froemke and Robert Willis Quackenbush, The spectrum of an equational class of

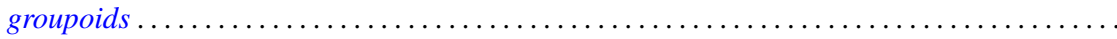

Barry J. Gardner, Radicals of supplementary semilattice sums of associative rings ...........

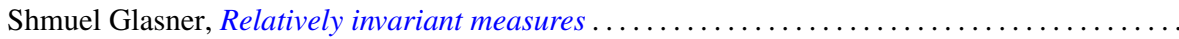

George Rudolph Gordh, Jr. and Sibe Mardesic, Characterizing local connectedness in inverse

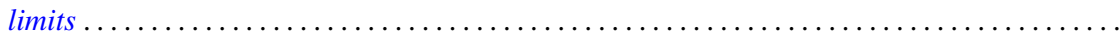

Siegfried Graf, On the existence of strong liftings in second countable topological spaces......

Stanley P. Gudder and D. Strawther, Orthogonally additive and orthogonally increasing

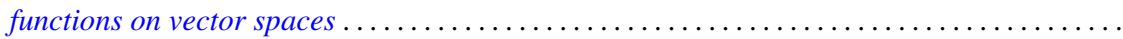

Darald Joe Hartfiel and Carlton James Maxson, A characterization of the maximal monoids and

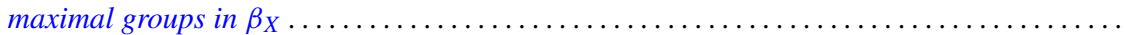

Robert E. Hartwig and S. Brent Morris, The universal flip matrix and the generalized faro-shuffle. .

William Emery Haver, Mappings between ANRs that are fine homotopy equivalences. .

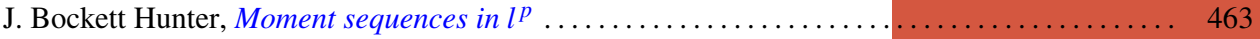

Barbara Jeffcott and William Thomas Spears, Semimodularity in the completion of a poset.... 467

Jerry Alan Johnson, A note on Banach spaces of Lipschitz functions . . . . . . . . . . . . 475

David W. Jonah and Bertram Manuel Schreiber, Transitive affine transformations on

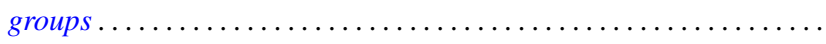

Karsten Juul, Some three-point subset properties connected with Menger's characterization of

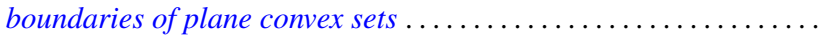

Ronald Brian Kirk, The Haar integral via non-standard analysis . . . . . . . . . . . . . 517

Justin Thomas Lloyd and William Smiley, On the group of permutations with countable

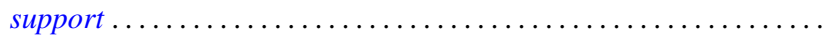

Erwin Lutwak, Dual mixed volumes .................................. 531

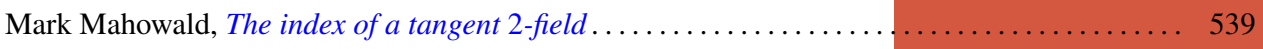

Keith Miller, Logarithmic convexity results for holomorphic semigroups . . . . . . . . . . . . 549

Paul Milnes, Extension of continuous functions on topological semigroups . . . . . . . . . . 553

Kenneth Clayton Pietz, Cauchy transforms and characteristic functions ................ 563

James Ted Rogers Jr., Whitney continua in the hyperspace $C(X) \ldots \ldots \ldots \ldots \ldots \ldots \ldots \ldots .569$

Jean-Marie G. Rolin, The inverse of a continuous additive functional . . . . . . . . . . . . 585

William Henry Ruckle, Absolutely divergent series and isomorphism of subspaces . ........ 605

Rolf Schneider, A measure of convexity for compact sets . ..................... 617

Alan Henry Schoenfeld, Continous measure-preserving maps onto Peano spaces .......... 627

V. Merriline Smith, Strongly superficial elements .......................... 643

Roger P. Ware, A note on quadratic forms over Pythagorean fields . . . . . . . . . . . . . . 651

Roger Allen Wiegand and Sylvia Wiegand, Finitely generated modules over Bezout rings . . . . 655

Martin Ziegler, A counterexample in the theory of definable automorphisms . . . . . . . . . 665 